\title{
Left Eye
}

National Cancer Institute

\section{Source}

National Cancer Institute. Left Eye. NCI Thesaurus. Code C119334.

The eye positioned in the left orbit. 